\section{Physical activity and cancer}

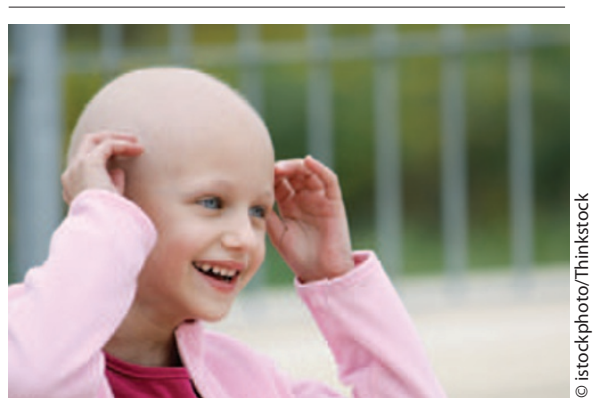

Studies in pediatric oncology have indicated a positive effect of physical activity on disease- and treatmentrelated side effects. Single studies demonstrate positive effects on the immune system, body composition, sleep, and activity levels. Results are very promising, but further highquality research is needed in order to establish evidence-based exercise recommendations for children with cancer. See page 366

\section{Biallelic mutations and lung injury}

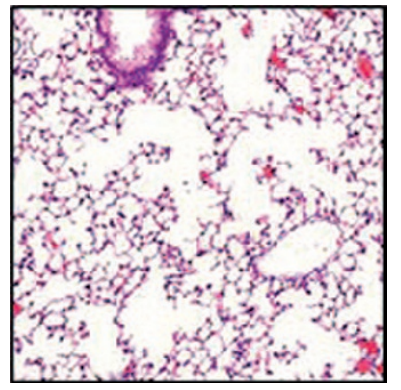

Heterozygous ABCA3 (ATP-bindingcassette subfamily A member 3 ) mutations are associated with neonatal respiratory complications. Using a murine model, HerberJonat and coinvestigators found that $A B C A 3$ haploinsufficiency results in a lung phenotype with altered biochemistry and mechanics. The inability to maintain a normal phosphatidylcholine-tophosphatidylglycerol ratio appears to play a key role. See page 384

\section{Infant cardiac surgery}
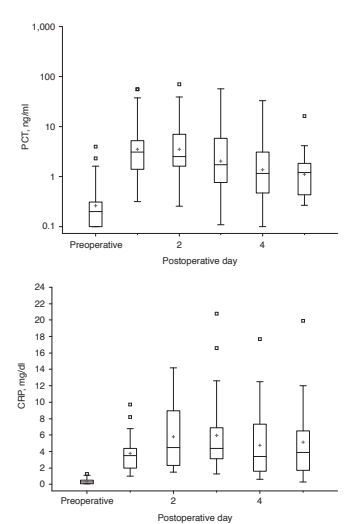

The utility of procalcitonin and C-reactive protein (CRP) as biomarkers of infection following cardiothoracic surgery in infants has not been well established. Davidson and colleagues conducted a cohort study to evaluate procalcitonin and CRP levels after such surgery. Procalcitonin appears to be independently associated with circulatory-support requirements at 72 hours postoperation, as well as with development of infection, and might have greater utility than CRP as a biomarker in this population.

See page 413
Pediatric obesity in China

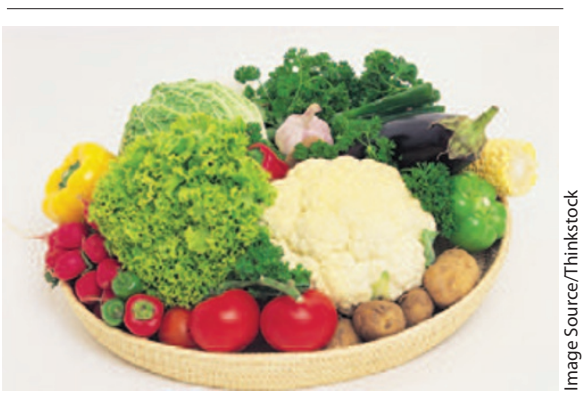

Guo et al. determined the prevalence of overweight and obesity, as well as the associated risk profiles, among children and adolescents in rural northeastern China. Using data from 4,094 participants, they found the prevalence of pediatric obesity in rural China to be relatively high. Risk profiles should be fully considered when planning prevention and treatment programs for obese children. See page 443

\section{APS 2013 Presidential Address}

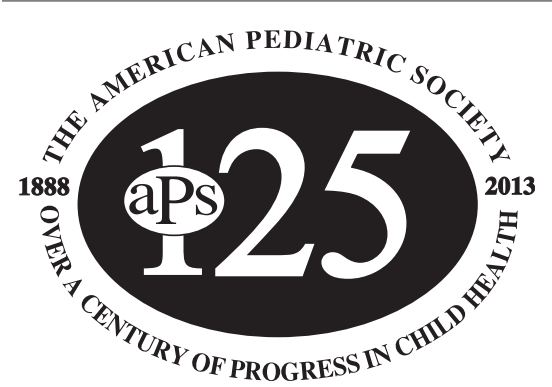

In her Presidential Address to the American Pediatric Society on its 125th anniversary, Barbara J. Stoll reflects on the organization's past as well as its future. See page 466 